\title{
Erratum to \\ Emerging Technologies for the 3D Analysis of Polymer Structures
}

Hiroshi Jinnai $^{1}(\bullet) \cdot$ Yukihiro Nishikawa $^{1} \cdot$ Takayuki Ikehara $^{2}$. Toshio Nishi ${ }^{3}$

${ }^{1}$ Department of Polymer Science and Engineering, Kyoto Institute of Technology, 606-8585 Kyoto, Japan

hjinnai@kit.ac.jp

${ }^{2}$ Department of Applied Chemistry, Faculty of Engineering, Kanagawa University, 3-27-1 Rokkakubashi, Kanagawa-ku, 221-8686 Yokohama, Japan

${ }^{3}$ Department of Organic and Polymeric Materials, School of Science and Engineering, Tokyo Institute of Technology, 2-12-1 Ohokayama, Meguro-ku, 152-8551 Tokyo, Japan

The online version of the original article can be found at http://dx.doi.org/10.1007/b95533

Unfortunately instead of the article "Emerging Technologies for the 3D Analysis of Polymer Structures" (H. Jinnai, Y. Nishikawa, T. Ikehara and T. Nishi) that is part of Advances in Polymer Science volume 170, the article "Coordination Polymerization of Dienes, Allenes, and Methylenecycloalkanes" (K. Osakada and D. Takeuchi) is listed in the online version. If you click on "entire document" the PDf file of the correct article will be shown. 